\title{
Origin and turbulence spreading of plasma blobs
}

\author{
P. Manz ${ }^{1,2}$, T.T. Ribeiro ${ }^{2}$, B.D. Scott $^{2}$, G. Birkenmeier ${ }^{1,2}$, D. Carralero ${ }^{2}$, \\ G. Fuchert ${ }^{3}$, S.H. Müller ${ }^{2}$, H.W. Müller ${ }^{2}$, U. Stroth ${ }^{2,1}$, E. Wolfrum ${ }^{2}$ \\ 1 Physik-Department E28, Technische Universität München, \\ James-Franck.Str. 1, Garching, Germany \\ 2 Max-Planck-Institut für Plasmaphysik, \\ Boltzmannstr. 2, Garching, Germany \\ 3 IJL, Université de Lorraine, CNRS (UMR 7198), \\ BP 40239, Vandoeuvre-lès-Nancy, France
}

(Dated: December 4, 2014)

\begin{abstract}
The formation of plasma blobs is studied by analyzing their trajectories in a gyrofluid simulation in the vicinity of the separatrix. Most blobs arise at the maximum radial electric field outside the separatrix. In general, blob generation is not bound to one particular radial position or instability. A simple model of turbulence spreading for the scrape-off layer is derived. The simulations show that the blob dynamics can be represented by turbulence spreading, which constitutes a substantial energy drive for far scrape-off layer turbulence and is a more suitable quantity to study blob generation compared to the skewness.
\end{abstract}




\section{INTRODUCTION}

Filamentary structures called blobs are dominating the transport in the region of open field lines, called scrape-off layer (SOL), and can seriously damage the vessel walls of magnetically confined fusion devices. Where the interchange forcing is responsible for the blob propagation $[1,2]$ the blob generation is not exclusively linked to the interchange instability [1-4]. But, also the drift-wave [5-7], the conducting wall instability [8] or the cooperative elliptic instability [9] have been reported to generate blobs. Furthermore not only the driving instability but also the magnetic and flow topology impact the blob formation. Whereas most driving instabilities act at the maximum normalized pressure gradient $-\nabla p_{e} / p_{e}[10]$,

blob generation has been observed at the shear layer $[4,11,12]$ or at the transition from closed to open field lines $[6,13]$. Blob formation in general is not bound to one particular position. Thus, which of the effects discussed above is most important also depends on the radial location considered. In general experimental results are biased by the positioning of the diagnostics or the reference position, if for example conditional averaging is used. A general mechanism explaining the ejection of blobs out of the unstable main plasma region into the far scrape-off layer, which is independent of the magnetic topology and driving instability is desirable. Such a mechanism can be provided by turbulence spreading for which a simplified model is developed in section. The present approach is independent of the underlying linear instability. Furthermore it constitutes a simple, self-consistent procedure to determine the position of blob formation and to quantify the nonlocal and nondiffusive behavior of transport. With this procedure it is possible to quantify which instability or which topological effect is most important for the blob formation.

Motivated by experiments on ASDEX-Upgrade [14], a detailed spatial temporal investigation of gyrofluid simulations at the last closed flux surface (LCFS) revealed a process, where blobs in and outside the confined region merge and thereby transfer density into the SOL [15]. This exemplifies the picture of turbulence spreading, where turbulent structures radially transport energy by merging and breaking up [16]. Even if turbulence spreading has been mostly studied in the context of zonal flow [16-18] and pinch [19] physics, it can be expected to be also important for the far SOL, where the mean gradients are found to be flatten out and hence cannot generate themselves any strong events in that region [20]. 


\section{GEMR SIMULATION}

In order to study the plasma blob birth simulations are needed, where blobs are generated self-consistent and are not seeded. Such a simulation is provided by the three-dimensional gyrofluid turbulence model GEMR [21] used here. Experimental and modeling results have been compared with reasonably good agreement [22-25]. The open field lines corresponding to the SOL are implemented by the boundary conditions via a perturbed Debye sheath current, such to retain the conducting wall instability (CWI) [26]. More details on the boundary conditions can be found in this Ref. [27]. Although being a $\delta$-f limited code the gradients evolve freely. The evolution of the gradients is necessary to capture the physics of turbulence spreading, which is a global phenomenon. However, the deviation from the preset background gradients has to be small. The parameters of the simulation analysed here are the same as in Refs. [15, 25], where blob dynamics in ASDEX Upgrade have been studied previously. The GEMR model includes, besides the standard interchange physics in tokamak geometry at the transition from closed to open field lines, also the drift-wave and the conducting wall instabilities. In sum it includes most effects which are believed to play an important role for the plasma blob dynamics which are three-dimensional [28], electromagnetic [29], finite ion temperature or finite Larmor radius [30-34] and shear flow (amongst others zonal flow and geodesic acoustic mode) effects [4, 11, 12].

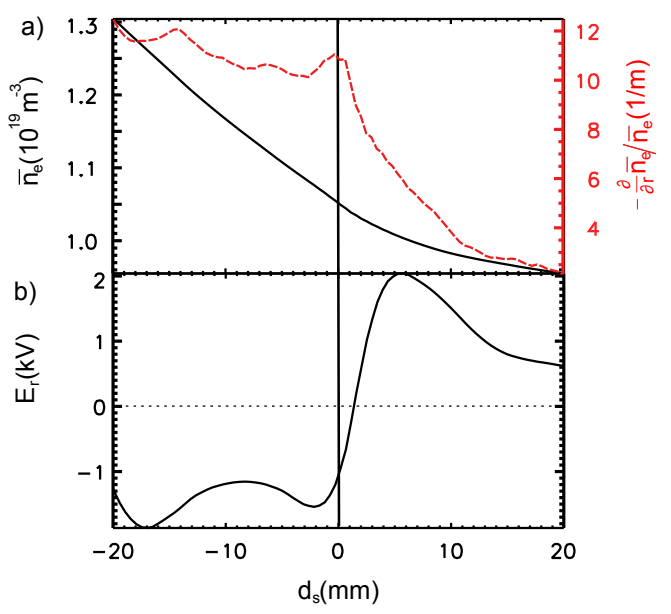

FIG. 1: (a) Background density and inverse background density gradient length, (b) background radial electric field around the LCFS. 


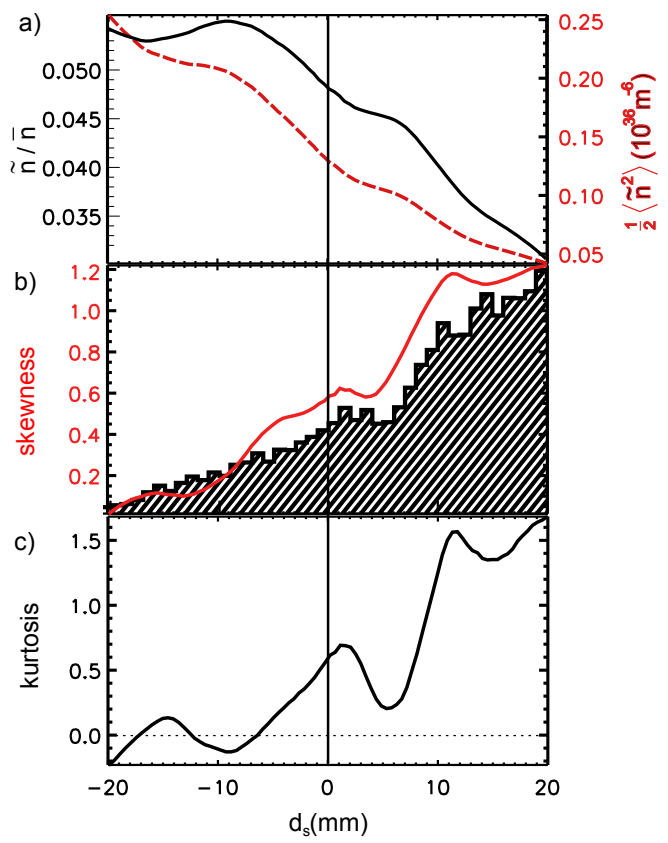

FIG. 2: Radial profiles of the (a) density fluctuation amplitude, (b) skewness of density fluctuations and radial distribution of blob trajectories, (c) excess kurtosis of density fluctuations.

\section{SKEWNESS AND BLOB TRAJECTORIES}

Blob birth is associated with the skewness of density fluctuations crossing zero $[1,13$, 14, 35]. The skewness and kurtosis profiles of the simulation are shown in Fig. 2. Both are small inside the confined region pointing to a non-intermittent character of the fluctuations. Across the separatrix both increase indicating a more intermittent behavior outside. The skewness takes very small values 1 to $2 \mathrm{~cm}$ inside the confined region. This region coincides with the maximum in $-\nabla n / n$ (Fig. 1a). Assuming the position of zero skewness is the position of the plasma blob generation, blobs would be generated about $2 \mathrm{~cm}$ inside the confined region. From that blobs are expected to be generated inside the confined region propagating across the LCFS into the SOL. As we will see in the following this interpretation is misleading.

The simulation provides the possibility to observe the blob generation directly. A snapshot of the density fluctuations is shown in Fig. 3a. Turbulent structures have a higher fluctuation amplitude inside the confined region as also shown by Fig. 2a. The reason is the stronger gradient. Outside the LCFS more positive than negative turbulent structures are 

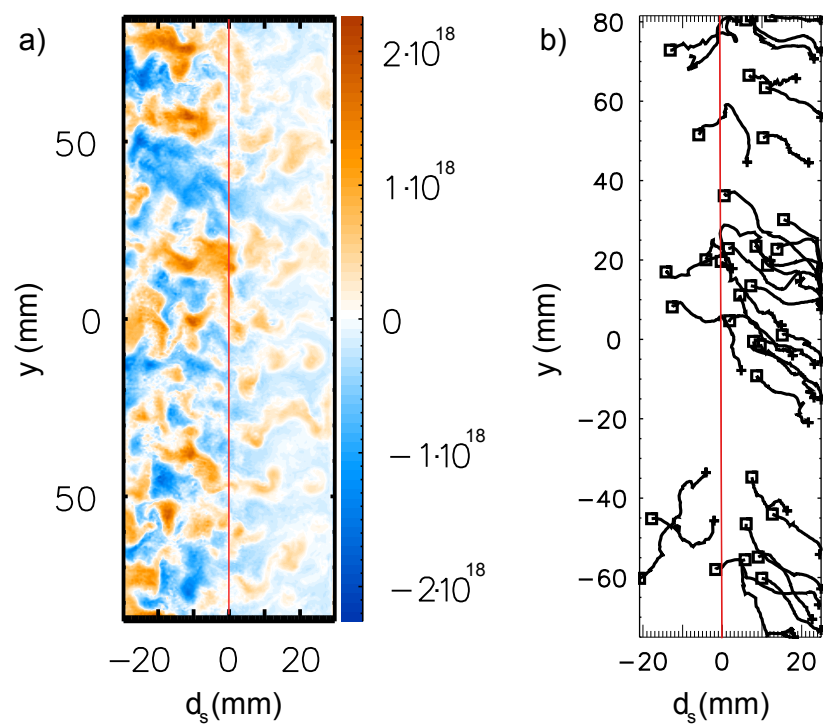

FIG. 3: (a) Snapshot of the fluctuating density. (b) Examples of blob trajectories.

observed, which can be also seen in the skewness (Fig. 2b). Positive density perturbations which appear coherent and isolated are blobs. Blobs are detected more frequently in the SOL. To investigate where blobs originate from they have to be defined first. Blob identification is done by three conditions. For every time step we identify blobs by two conditions. First we define blobs as positive density fluctuations, which exceed the standard deviation by a factor of 2.5. The radial local value of the standard deviation is used here, since it is usually used in experimental investigations. Second the blob has to fulfill this condition over a spatial extent of a least 11 points of the simulation grid $\left(\approx 8 \rho_{s} \approx 5 \mathrm{~mm}\right)$. After possible blobs have been identified for every time step these are tracked using the algorithm of Ref. [36]. Only structures which can be tracked for at least 50 time steps $(\approx 25 \mu \mathrm{s})$ are considered as blobs. That is the third condition. The radial distribution of blob trajectories is inserted in Fig. 2b, showing a good agreement of the occurrence of the detected blobs with the skewness. The radial position of the first occurrence of a structure satisfying these conditions is the plasma blob birth location. The last occurrence will be defined as the blob death.

Examples for blob trajectories are shown in Fig. 3b. Blobs are generated over the entire domain. This observation is also shown in the blob birth statistics in Fig. 4a. Also the blobs show a pronounced poloidal asymmetry. Most of the blobs are detected above the midplane pointing to the fact that the magnetic configuration impacts the local dynamics 

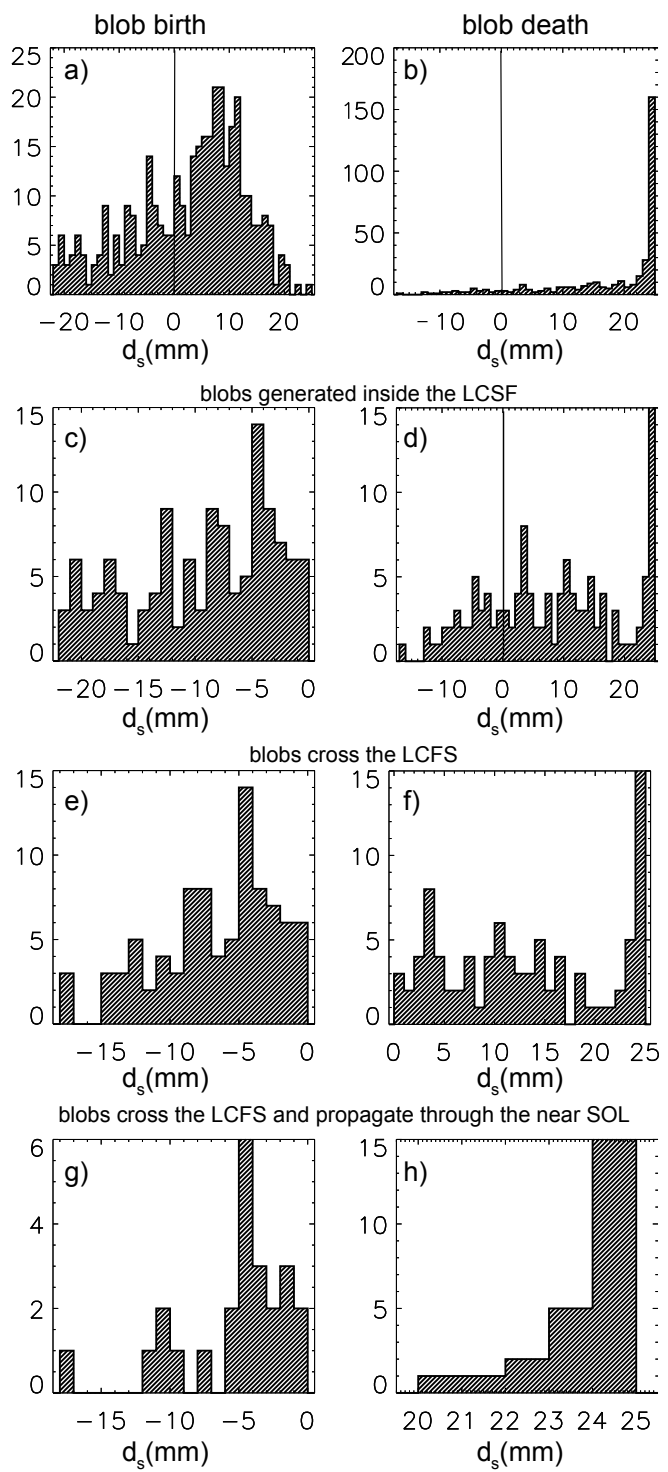

FIG. 4: Statistics of the (a,c,e,g) birth and $(b, d, f, h)$ death location of the detected blobs also under selective conditions: only blob which are generated inside the LCFS (c,d), which cross the seperatrix $(e, f)$ and which are generated inside the LCFS and propagate into the far SOL $(g, h)$ are considered.

similar to Ref. [37]. Some blobs form inside the confined region, where the background gradients are high, and propagate outwards (Fig. 4c). Most of them cross the separatrix (Fig. 4d). These are usually generated close to the LCFS (Fig. 4e). By propagating across the LCFS they change the poloidal propagation direction (Fig. 3b). In the confined region blobs propagate in the electron diamagnetic direction, in the SOL in the ion diamagnetic 
direction. A remarkable fraction of blobs vanishes at the shear layer by either being torn apart or strained out [38], but most blobs reach the boundary of the simulation.

\section{CONDUCTING WALL INSTABILITY}

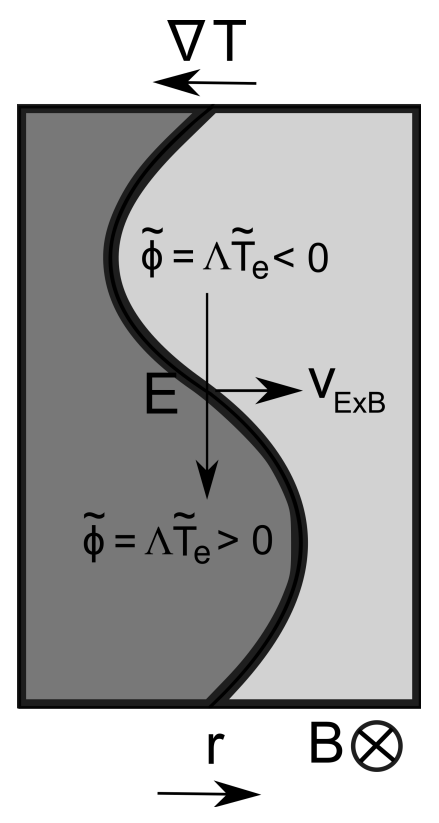

FIG. 5: Dark regions indicate higher electron temperature. Electron temperature fluctuations induce in-phase potential fluctuations via the floating potential $\Lambda$. The resulting $E \times B$ drift leads to a propagation in the electron diamagnetic direction.

However, as seen in Fig. 4a most of the blobs do not originate from the confined region and are generated $7 \mathrm{~mm}$ outside the LCFS, where the poloidal velocity (proportional to the radial electric field shown in Fig. 1a) takes its maximum. Most of these blobs propagate outside the simulation domain. The radial electric field in the SOL is needed to maintain quasi-neutrality, where the potential limits electron end losses to the wall. The resulting poloidal velocity gives rise to the CWI [8]. The basic mechanism of the CWI is shown in Fig. 5. The CWI is driven by electron temperature gradient. Fluctuations of the parallel current are induced by electron temperature fluctuations via the Bohm criterion [39]. Then the fluctuations in the parallel current couple electron temperature and plasma potential fluctuations. Similar to the common drift-wave instability the thermodynamic fluctuations (for the CWI it is the electron temperature, for the drift-wave it is the density) are in phase 
to the potential fluctuations. In this case the CWI is stable. But as for the drift-wave any restriction on the parallel electron dynamics as collisions will drive the CWI unstable. In general the linear growth rate of the CWI is of the same order as that of the interchange instability [39]. As seen in a previous study [25] electron temperature fluctuations are strong enough to dominate floating potential fluctuations close to the separatrix. At ASDEX Upgrade these fluctuations are non-negligible in general and the dominance of the CWI for ASDEX Upgrade parameters is consistent with a previous study [25].

In general it seems that the transition from drift-wave dominated turbulence to interchange dominated turbulence corresponds to the main generation location of blobs $[6,7]$. The CWI is a drift-wave-like instability and extents this region a few millimeters into the SOL.

Because of the definition of a blob the birth location depends on the local variation of the standard deviation. Structures generated in the confined region below the critical standard deviation are not considered to be blobs. As these structures propagate outward they may match the blob criterion at a position in the SOL, which is regarded as the blob birth location even thought the structure is not generated at this position by a linear instability. A previous study with GEMR shows that the fluctuations at the high field side disappear, if it is disconnected from the low field side by limiters [27]. Although the high field side is sheath connected in this configuration and therefore the situation is very similar for the CWI, the fluctuation levels are very low, thus the turbulence is driven by energy input from the confined region, which appear on the low field side. Blob generation appears to be a nonlocal phenomenon.
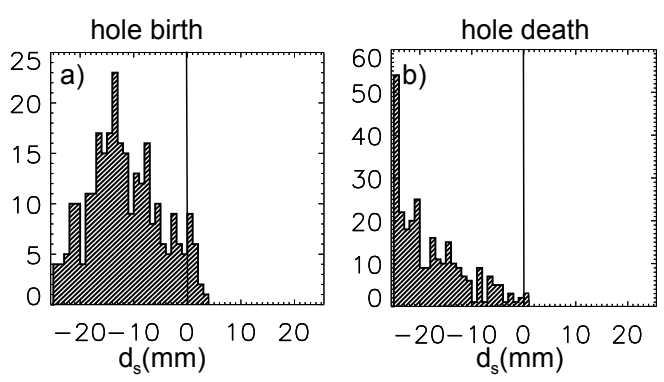

FIG. 6: Statistics of the (a) birth and (b) death location of the detected holes. 


\section{PLASMA HOLES}

As blob ejection is often associated with the generation of plasma holes $[1,13,14]$ their trajectories are analyzed, too. Since plasma holes are not as pronounced as plasma blobs we have to relax the conditions by defining a plasma hole as a structure going below the limit of two times the negative standard deviation for 30 frames $(\approx 15 \mu \mathrm{s})$. The birth/death statistics of plasma holes is shown in Fig. 6. The generation of holes is restricted to the confined region. Holes have the tendency to propagate inwards. The restriction of holes to the confined plasma region could be an interesting observation in respect to the magnetization interchange instability [40]. However, for the time being we leave a detailed investigation of the electro-magnetic behavior for future work.

\section{TURBULENCE SPREADING}

Finally the energetics is investigated. A simple so-called $K-\epsilon$ model similar to that of Ref. [18] is derived to investigate turbulence spreading. Let us consider the radial convective part of the continuity equation only

$$
\frac{\partial n}{\partial t}=-v_{r} \frac{\partial}{\partial r} n
$$

neglecting damping and cross-field coupling. Fluctuations are defined by taking the difference to the zonal and time average $\langle\cdot\rangle=\int d y d s d t$ as the mean quantity $\tilde{n}=n-\langle n\rangle$. For simplicity the radial background flow is neglected $\left\langle\tilde{v}_{r}\right\rangle=0$ and the plasma turbulence is incompressible in the perpendicular plane, therefore $\partial_{r} v_{r}$ cancels out with its binormal counterpart. The evolution of the mean density neglecting sources, sinks and dissipation of simplicity is given by

$$
\frac{\partial\langle n\rangle}{\partial t}=-\left\langle\tilde{v}_{r} \frac{\partial}{\partial r} \tilde{n}\right\rangle=-\frac{\partial}{\partial r}\left\langle\tilde{v}_{r} \tilde{n}\right\rangle
$$

we see that the background density is effected by the divergence of the turbulent transport $\Gamma=\left\langle\tilde{v}_{r} \tilde{n}\right\rangle$. As a divergence it conserves particles except at the boundary terms. The evolution of the background free energy is

$$
\frac{1}{2} \frac{\partial\langle n\rangle^{2}}{\partial t}=-\langle n\rangle \frac{\partial}{\partial r}\left\langle\tilde{v}_{r} \tilde{n}\right\rangle=-\frac{\partial}{\partial r}\left(\langle n\rangle\left\langle\tilde{v}_{r} \tilde{n}\right\rangle\right)+\left\langle\frac{\partial n}{\partial r}\right\rangle\left\langle\tilde{v}_{r} \tilde{n}\right\rangle
$$


The total free energy $(1 / 2)(\langle n\rangle+\tilde{n})^{2}$ evolution is given by

$$
\begin{aligned}
\frac{1}{2} \frac{\partial\left\langle(\langle n\rangle+\tilde{n})^{2}\right\rangle}{\partial t} & =-\left\langle(\langle n\rangle+\tilde{n}) \tilde{v}_{r} \frac{\partial}{\partial r}(\langle n\rangle+\tilde{n})\right\rangle \\
& =-\langle n\rangle \frac{\partial}{\partial r}\left\langle\tilde{v}_{r} \tilde{n}\right\rangle-\left\langle\frac{\partial n}{\partial r}\right\rangle\left\langle\tilde{v}_{r} \tilde{n}\right\rangle-\left\langle\tilde{n} \tilde{v}_{r} \frac{\partial \tilde{n}}{\partial r}\right\rangle .
\end{aligned}
$$

Subtracting the mean evolution gives the evolution of the turbulent free energy

$$
\frac{1}{2} \frac{\partial\left\langle\tilde{n}^{2}\right\rangle}{\partial t}=-\left\langle\frac{\partial n}{\partial r}\right\rangle\left\langle\tilde{v}_{r} \tilde{n}\right\rangle-\left\langle\tilde{n} \tilde{v}_{r} \frac{\partial \tilde{n}}{\partial r}\right\rangle
$$

using $\partial_{r}\left\langle\tilde{v}_{r} \tilde{n} \tilde{n}\right\rangle=2\left\langle\tilde{v}_{r} \tilde{n} \partial_{r} \tilde{n}\right\rangle$ results in

$$
\frac{1}{2} \frac{\partial\left\langle\tilde{n}^{2}\right\rangle}{\partial t}=-\left\langle\frac{\partial n}{\partial r}\right\rangle\left\langle\tilde{v}_{r} \tilde{n}\right\rangle-\frac{1}{2} \frac{\partial}{\partial r}\left\langle\tilde{v}_{r} \tilde{n}^{2}\right\rangle
$$

The first term of this equation appears with a different sign in the evolution of the background free energy (last term in Eq. (3)) and therefore constitutes a transfer term, which transfers the free energy from the background to the turbulent field. Where transport is responsible for the main loss of particles, it is the main drive of turbulence. We define the local drive of the turbulence by

$$
\mathcal{D}=-\left\langle\frac{\partial n}{\partial r}\right\rangle\left\langle\tilde{v}_{r} \tilde{n}\right\rangle
$$

The second term is a divergence and preferentially transports the fluctuation amplitude from unstable to stable regions. This is the turbulence spreading term

$$
\mathcal{S}=-\frac{1}{2} \frac{\partial}{\partial r}\left\langle\tilde{v}_{r} \tilde{n}^{2}\right\rangle
$$

Next we use the 3D GEMR simulation already analyzed before to estimate the quantities discussed above. The present analysis is restricted to the same 2D plane at the low field side midplane we have already analyzed in Figs. 1,2,3,4,6, where blobs are prominent. The region considered here is similar to those of a $2 \mathrm{D}$ simulation or experimental data from gas-puff imaging at the outboard midplane. It can be easily applied in those configurations. The average used in the following is $\langle\cdot\rangle=\int d y d t$. For $1 \mathrm{D}$ probe measurements $\langle\cdot\rangle=\int d t$ may be used. Figure 7 compares the rates of turbulent drive by the background gradient

$$
\omega_{\mathcal{D}}=\frac{-\left\langle\frac{\partial n}{\partial r}\right\rangle\left\langle\tilde{v}_{r} \tilde{n}\right\rangle}{\frac{1}{2}\left\langle\tilde{n}^{2}\right\rangle}
$$




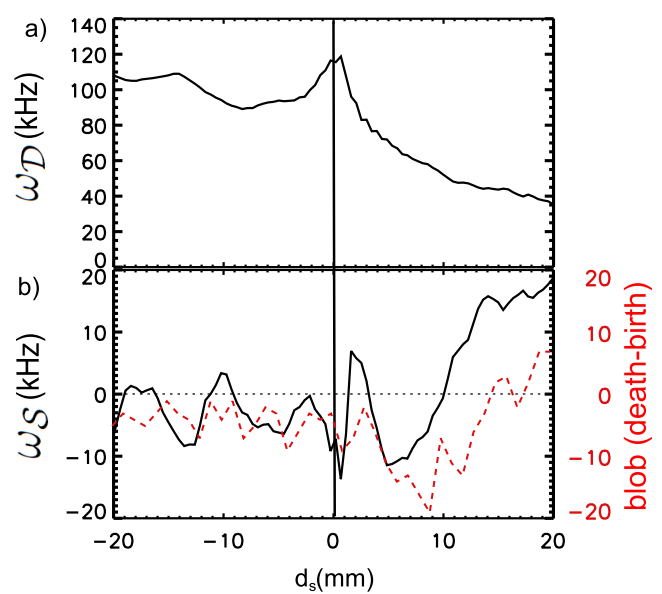

FIG. 7: (a) Energetic drive by the background gradient and (b) turbulence spreading of density fluctuations (black solid line). The red dashed line in (b) shows the number of blobs that died reduced by the number of blobs born.

with the rate of turbulence spreading

$$
\omega_{\mathcal{S}}=\frac{-\frac{1}{2} \frac{\partial}{\partial r}\left\langle\tilde{v}_{r} \tilde{n}^{2}\right\rangle}{\frac{1}{2}\left\langle\tilde{n}^{2}\right\rangle},
$$

both normalized to the local fluctuation amplitude. The normalized turbulent drive as shown in Fig. 7a is interpreted as the local linear effective growth rate and the normalized turbulence spreading as shown in Fig. 7b as the nonlocal nonlinear growth rate. The local linear effective growth rate is higher in the confined region and peaks at the LCFS. The turbulent drive is very similar to the inverse background gradient (Fig. 1a). Most of the blobs are not generated at this position (Fig. 4). At the LCFS a transfer of free energy by turbulence spreading from the confined region into the SOL consistent is observed. Compared to the linear growth rate this energy transfer is small (10\%). This corresponds to the cascade-like picture of turbulence spreading [16] recently investigated at the shear layer close to the LCFS in simulations and experiments [15].

In the SOL turbulence spreading transports the free energy from the region $7 \mathrm{~mm}$ outside the LCFS into the far SOL. The generation location of the turbulent intensity transport corresponds to the position, where the radial electric field exhibits its maximum (Fig. 1a) and the blobs are generated. The blob source is given by the number of blobs born reduced by the their deaths. The negative of the blob source (red dashed line in Fig. 7b) roughly follows the turbulence spreading (black solid line in Fig. 7a). Turbulence spreading connects 
blob sources and sinks. The analysis of turbulence spreading indicate a blob birth location of $7 \mathrm{~mm}$ outside the LCFS which is pretty close to the actual position at $9 \mathrm{~mm}$, whereas the condition of crossing zero skewness (Fig. 2b) would predict the blob birth region at $20 \mathrm{~mm}$ inside the LCFS. Turbulence spreading provide a more suitable diagnostic for the blob birth location than the skewness alone. Spreading becomes stronger further out and reaches half of the local drive $2 \mathrm{~cm}$ outside the LCFS (black solid line in Fig. 7a). It can be therefore expected that turbulence spreading is the main drive in the far SOL. Turbulence spreading in the SOL is therefore mainly carried by the blobs and not by the cascade-like interaction. It could be demonstrated that GEMR as a global code is capable to study the basic effects of turbulence spreading. Since GEMR is a $\delta$-f limited code it would be interesting to compare with a full- $f$ gyrofluid code like that in Ref. [41].

\section{CONCLUSION}

In conclusion, blobs are not generated at one particular position. They are generated at different radial positions due to different instabilities. In the present simulation blob formation takes place at the maximum of the radial electric field outside the separatrix. At this position the conducting wall mode exhibit its maximum growth. However, it is not the linear instability that is responsible for the blob formation. The blob formation coincides with turbulent spreading and not with the linear drive. Turbulence spreading plays an important role for the SOL. It provides a more suitable diagnostic for blob generation compared to the skewness alone and it constitutes an energy exchange mechanism between the confined plasma with the SOL and a substantial energy drive for fluctuations in the far SOL. The present theory can be easily investigated with the standard transport diagnostics (probes and GPI) in the SOL.

[1] D. A. D’Ippolito, J. R. Myra, and S. J. Zweben, Phys. Plasmas 18, 060501 (2011).

[2] S. Krasheninnikov, Phys. Lett. A 283, 368 (2001).

[3] N. Bisai, A. Das, S. Deshpande, P. K. Kaw, A. Sen, and R. Singh, Phys. Plasmas 12, 102515 (2005). 
[4] I. Furno, B. Labit, M. Podesta, A. Fasoli, S. H. Müller, P. Poli, F. M.and Ricci, C. Theiler, S. Brunner, A. Diallo, and J. Graves, Phys. Rev. Lett. 100, 055004 (2008).

[5] T. Windisch, O. Grulke, and T. Klinger, Phys. Plasmas 13, 122303 (2006).

[6] T. Happel, F. Greiner, N. Mahdizadeh, B. Nold, M. Ramisch, and U. Stroth, Phys. Rev. Lett. 102, 255001 (2009).

[7] G. Fuchert, G. Birkenmeier, B. Nold, M. Ramisch, and U. Stroth, Plasma Phys. \& Controlled Fusion 55, 125002 (2013).

[8] H. L. Berk, R. H. Cohen, D. D. Ryutov, Y. A. Tsidulko, and X. Q. Xu, Nucl. Fusion 33, 263 (1993).

[9] P. Manz, M. Xu, S. H. Müller, N. Fedorczak, S. C. Thakur, J. H. Yu, and G. R. Tynan, Phys. Rev. Lett. 107, 195004 (2011).

[10] J. R. Myra, D. A. D’Ippolito, D. P. Stotler, S. J. Zweben, B. P. LeBlanc, J. E. Menard, R. J. Maqueda, and J. Boedo, Physics of Plasmas 13, 092509 (2006).

[11] G. S. Xu, V. Naulin, W. Fundamenski, C. Hidalgo, J. A. Alonso, C. Silva, B. Gonalves, A. H. Nielsen, J. J. Rasmussen, S. Krasheninnikov, et al., Nuclear Fusion 49, 092002 (2009).

[12] J. Cheng et al., Phys. Rev. Lett. 110, 265002 (2013).

[13] J. A. Boedo, D. L. Rudakov, R. A. Moyer, G. R. McKee, R. J. Colchin, M. J. Schaffer, P. G. Stangeby, W. P. West, S. L. Allen, T. E. Evans, et al., Physics of Plasmas 10, 1670 (2003).

[14] B. Nold, G. D. Conway, T. Happel, H. Müller, M. Ramisch, V. Rohde, U. Stroth, and ASDEX Upgrade Team, Plasma Phys. \& Controlled Fusion 52, 065005 (2010).

[15] B. Nold, P. Manz, T. T. Ribeiro, G. Fuchert, G. Birkenmeier, H. W. Müller, M. Ramisch, B. D. Scott, and U. Stroth, Phys. Plasmas 21, 102304 (2014).

[16] Ö. D. Gürcan, P. H. Diamond, T. S. Hahm, and Z. Lin, Phys. Plasmas 12, 032303 (2005).

[17] T. Estrada, C. Hidalgo, T. Happel, and P. H. Diamond, Phys. Rev. Lett. 107, 245004 (2011).

[18] P. Manz, M. Xu, N. Fedorczak, S. C. Thakur, and G. R. Tynan, Phys. Plasmas 19, 012309 (2012).

[19] V. Naulin, A. H. Nielsen, and J. J. Rasmussen, Phys. Plasmas 12, 122306 (2005).

[20] P. Ghendrih, Y. Sarazin, G. Ciraolo, G. Darmet, X. Garbet, V. Grandgirard, P. Tamain, S. Benkadda, and P. Beyer, J. Nucl. Mat. 363-365, 581 (2007).

[21] B. D. Scott, Contrib. Plasma Phys. 46, 714 (2006).

[22] S. J. Zweben, B. D. Scott, J. L. Terry, B. LaBombard, J. W. Hughes, and D. P. Stotler, Phys. 
Plasmas 16, 082505 (2009).

[23] F. Gennrich and A. Kendl, Plasma Phys. \& Controlled Fusion 54, 015012 (2012).

[24] M. Kočan, F. P. Gennrich, A. Kendl, H. W. Müller, and ASDEX Upgrade Team, Plasma Phys. \& Controlled Fusion 54, 085009 (2012).

[25] B. Nold, T. T. Ribeiro, M. Ramisch, Z. Huang, H. W. Müller, B. D. Scott, U. Stroth, and ASDEX Upgrade Team, New. J. Phys. 14, 063022 (2012).

[26] T. T. Ribeiro and B. Scott, Plasma Phys. \& Controlled Fusion 47, 1657 (2005).

[27] T. T. Ribeiro and B. Scott, Plasma Phys. \& Controlled Fusion 50, 055007 (2008).

[28] J. R. Angus, M. V. Umansky, and S. I. Krasheninnikov, Phys. Rev. Lett. 108, 215002 (2012).

[29] G. S. Xu, V. Naulin, W. Fundamenski, J. J. Rasmussen, A. H. Nielsen, and B. N. Wan, Phys. Plasmas 17, 022501 (2010).

[30] N. Bisai, R. Singh, and P. K. Kaw, Phys. Plasmas 19, 052509 (2012).

[31] P. Manz, D. Carralero, G. Birkenmeier, H. W. Müller, S. H. Müller, G. Fuchert, B. D. Scott, and U. Stroth, Phys. Plasmas 20, 102307 (2013).

[32] J. Madsen, O. E. Garcia, J. S. Larsen, V. Naulin, A. H. Nielsen, and J. J. Rasmussen, Phys. Plasmas 18, 112504 (2011).

[33] G. Birkenmeier, F. M. Laggner, M. Willensdorfer, T. Kobayashi, P. Manz, E. Wolfrum, D. Carralero, R. Fischer, B. Sieglin, G. Fuchert, et al., Plasma Phys. \& Controlled Fusion 56, 075019 (2014).

[34] G. Fuchert, G. Birkenmeier, D. Carralero, T. Lunt, P. Manz, H. W. Müller, B. Nold, M. Ramisch, V. Rohde, U. Stroth, et al., Plasma Phys. \& Controlled Fusion 56, 125001 (2014).

[35] N. Yan, A. H. Nielsen, G. S. Xu, V. Naulin, J. Rasmussen, J. Madsen, H. Q. Wang, S. C. Liu, W. Zhang, L. Wang, et al., Plasma Phys. \& Controlled Fusion 55, 115007 (2013).

[36] J. C. Crocker and D. G. Grier, J. Colloidal Interface Sci. 179, 298 (1996).

[37] G. Birkenmeier, M. Ramisch, P. Manz, B. Nold, and U. Stroth, Phys. Rev. Lett. 107, 025001 (2011).

[38] P. Manz, M. Ramisch, and U. Stroth, Phys. Rev. Lett. 103, 165004 (2009).

[39] Y. Sarazin, P. Ghendrih, G. Attuel, C. Clement, X. Garbet, V. Grandgirand, M. Ottaviani, S. Benkadda, P. Beyer, N. Bian, et al., J. Nucl. Mater. 313-316, 796 (2003).

[40] E. Solano and R. D. Hazeltine, Nucl. Fusion 52, 114017 (2012). 
[41] M. Wiesenberger, J. Madsen, and A. Kendl, Phys. Plasmas 21, 092301 (2014). 